RICYDE. Revista Internacional de Ciencias del Deporte doi: $10.5232 /$ ricyde

Rev. int. cienc. deporte

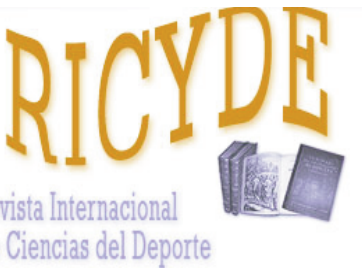

RICYDE. Revista Internacional de Ciencias del Deporte VOLUMEN XIII - AÑO XIII

Páginas:314-330 ISSN:1885-3137

Número 50 - Octubre - 2017

\title{
El control de la violencia deportiva en el fútbol profesional español (1985-1995) Sporting violence curving in Spanish professional football (1985-1995)
}

\author{
Carlos García-Martí, Javier Durán-González, Maite Gómez-López \\ Universidad Politécnica de Madrid. España
}

\begin{abstract}
Resumen
El objetivo de este artículo es analizar el descenso de la violencia permitida y ejercida en el terreno de juego a principios de la década de los noventa en el fútbol profesional español. Para ello se ha procedido a desarrollar una investigación cualitativa que incluye 31 entrevistas, que incluyen a once futbolistas profesionales, once entrenadores, cinco periodistas y tres árbitros en activo en el periodo estudiado y un historiador. La selección de la muestra se llevó a cabo a través de un muestreo de conveniencia y una técnica de captación de bola de nieve. Estas entrevistas muestran que la violencia era parte de la ética profesional y que su reducción se llevó a cabo a través de una doble coerción, el aumento del rigor disciplinario y la denuncia de los medios de comunicación. Esta pacificación formó parte de la imposición del nuevo discurso hegemónico del fútbol espectáculo ligado a los cambios en el modelo de negocio. En consecuencia, la identidad de los futbolistas estuvo menos ligada a una masculinidad tradicional, y debieron desarrollar nuevas habilidades de mayor peso cognitivo para adaptarse.
\end{abstract}

Palabras clave: fútbol; España, violencia; medios de comunicación; masculinidad.

\begin{abstract}
The objective of this article is to analyze how exerted and allowed violence was reduced in Spanish professional football in the nineties decade. In order to do so, a qualitative research was undertaken carrying on 31 in-depth interviews, including eleven footballers, eleven football managers, five journalists and three referees, all active during the period under study, and one historian. A theoretical sample was designed, and participants recruited through a snow ball technique. The interviews show that violence was part of the professional ethics, and that its reduction was made possible through the hardening of the discipline and the pressure from the media. This pacification was part of a new discourse of show business linked to the commodification process in place. Footballers' identity was in turn less defined by a traditional masculinity, having to develop new cognitive skills to deal with the new context.
\end{abstract}

Key words: football; Spain; violence; media; masculinity. 
García-Martí, C.; Durán-González, J., y Gómez-López, M. (2017). El control de la violencia deportiva en el fútbol profesional español (1985-1995). RICYDE. Revista internacional de ciencias del deporte. 50(13), 322-338. https://doi.org/10.5232/ricyde2017.05001

\section{Introducción}

$\mathrm{E}$ n el ámbito sociológico el estudio de los deportistas profesionales es un campo en pleno desarrollo y del máximo interés, puesto que se trata de los principales protagonistas de aquello que conocemos como deporte espectáculo, una de las instituciones centrales de la cultura popular contemporánea. Su estudio como trabajadores permite, por un lado, salir del encantamiento espontáneo que su proyección mediática genera (Bertrand, 2009), al mismo tiempo que se genera un conocimiento práctico de utilidad para los propios profesionales sobre el funcionamiento de su mundo social. Por ello, este artículo pretende arrojar luz sobre el fenómeno centrándose en la relación de los futbolistas con la violencia en el terreno de juego como parte de su profesión, desde una perspectiva sociohistórica.

La literatura sociológica británica ha descrito a la cultura profesional del fútbol como centrada en el conocimiento práctico, proveniente de la experiencia y que rechaza las nociones teóricas (Kelly, 2008), en lo que se asemejaría a una profesión artesanal (Day, 2011). En cuanto a la violencia propiamente dicha, los estudios señalan que, por un lado, las relaciones entre futbolistas y entrenadores se caracterizan por el uso de la intimidación y de la violencia simbólica dentro de una relación de autoridad tradicional en el sentido weberiano (Kelly y Waddington, 2006), caracterizada por la discrecionalidad en el ejercicio del poder y la falta de controles formales. Dentro de esa relación, los futbolistas entienden que su posición en el equipo depende fundamentalmente de mostrar una buena actitud, consistente por un lado en la lealtad y subordinación al entrenador, pero también por un compromiso en el campo que incluye una enorme agresividad en las disputas por el balón (Roderick, 2003). Todo ello formaría parte de una masculinidad agresiva (Parker, 1996; Roderick, Waddington, y Parker, 2000) que incluye también soportar el dolor o jugar lesionados por el bien del equipo (Parker 1996; Roderick 2003; Bertrand 2009; Llopis 2008). Igualmente existe una cultura de la hipercompetitividad, debido a la incertidumbre profesional, y de una defensa de la agresividad que comienza en el periodo formativo (Bertrand, 2009; Parker, 1996), rasgos todos ellos que parecen ser constitutivos de la profesión desde sus inicios históricos (Harding \& Taylor, 2003).

Muchos de estos rasgos han sido constatados en España por Llopis (2008) tanto en las actitudes del público como de los responsables de la formación de los jóvenes futbolistas, que les inculcan la agresividad y las formas más tradicionales de masculinidad, lo que le ha hecho afirmar que el fútbol es un espacio de reproducción de la masculinidad hegemónica. Este concepto proveniente de los estudios de género (Carrigan, Connell, y Lee, 1985; Connell y Messerschmidt, 2015) sostiene que la superioridad masculina es el resultado de un estado de fuerzas, y que la masculinidad hegemónica sería la forma ideal, discursiva, de la masculinidad que es movilizada para mantener los privilegios de los hombres, sigan o no los patrones de esta masculinidad en su vida cotidiana. En el campo del deporte, Messner (1988, 1989, 1990) entiende que éste es un espacio refugio para los hombres frente al crecimiento del poder femenino y la pérdida de poder que supone la industrialización y burocratización de la sociedad. Así, los hombres movilizan el ideal deportivo masculino para intentar sostener una superioridad biológica frente a la mujer que han perdido en muchos otros ámbitos, o como sostiene uno de sus entrevistados que "una mujer puede hacer el mismo trabajo que yo o incluso ser mi jefe, pero que me aspen si puede salir al campo de fútbol y soportar un golpe de Ronnie Lott [jugador de fútbol americano]" (Messner, 1989, p. 79). 
García-Martí, C.; Durán-González, J., y Gómez-López, M. (2017). El control de la violencia deportiva en el fútbol profesional español (1985-1995). RICYDE. Revista internacional de ciencias del deporte. 50(13), 322-338. https://doi.org/10.5232/ricyde2017.05001

Mientras que, por tanto, el deporte aparece como el último refugio de la masculinidad más tradicional, las ideas de la Escuela de Leicester apuntan a una interpretación alternativa. Como es sabido, para Elias y Dunning (E Dunning y Sheard, 2005; Elias y Dunning, 1966, 1992) el deporte ha sufrido un fuerte proceso civilizador que ha incluido una fuerte reducción de la violencia, a través del desarrollo de reglamentos y de autoridades reconocidas. Los deportistas profesionales ejercerían fundamentalmente una violencia puramente instrumental, con la intención de obtener una ventaja deportiva y profesional aun cuando se sitúen fuera del reglamento, y las explosiones de violencia emotiva o irracional serían propias del público, y únicamente de los sectores de éste menos expuestos a la espiral civilizatoria, en concreto los sectores de la clase obrera menos integrados. Esta interpretación ha sufrido tanto la crítica teórica de aquellos que rechazan esta estratificación de la clase obrera (Best, 2010), así como de la evidencia empírica que niega que los hooligans provengan de sectores excluidos socialmente (Giulianotti, 2000), pero no puede negarse sin embargo que el desarrollo de los deportes modernos supuso un descenso de la violencia permitida respecto a sus antecedentes preindustriales.

En esta descripción debemos añadir un último factor, como son los cambios en el espectáculo deportivo que han tenido lugar desde los años ochenta, y que en el caso del fútbol hacen hablar del nuevo negocio del fútbol - new football business - fundamentado en la televisión y los patrocinios y por tanto interesado en la espectacularidad y la búsqueda de audiencias masivas y variadas (Giulianotti, 2000; King, 1997; Sandvoss, 2003). Esta evolución es importante porque uno de los intereses del nuevo fútbol es el de atraer a públicos hasta entonces menos interesados por el fenómeno, como las nuevas clases medias profesionales y el público femenino, que en el caso inglés ha tenido como resultado la exclusión económica de amplias capas de la clase obrera del consumo del fútbol, aquellas precisamente que Dunning consideraba más proclives a enaltecer o celebrar la violencia.

En el caso español, este proceso comienza con la conversión de los clubes en Sociedades Anónimas Deportivas por la Ley del Deporte de 1990 (García, Gómez, y Durán, 2016) y por la aparición de los nuevos operadores privados, especialmente Canal+, que van a multiplicar los ingresos de los clubes por retransmisiones (Bonaut, 2010). Coincide también con la identificación por la opinión pública del vandalismo en el fútbol como problema social (Durán, 1996), que alcanzó su cénit con el fallecimiento de un menor por el impacto de una bengala el 15 de marzo de 1992 en el estadio del Espanyol, por lo que se conoció como la tragedia de Sarriá. De hecho, semanas después se pondría en marcha la Comisión Nacional Antiviolencia prevista por la Ley del Deporte de 1990 comenzando la lucha por la erradicación de la violencia en las gradas. También la FIFA comienza a tomar las primeras medidas para reducir la violencia en el campo dentro de un programa de relanzamiento del espectáculo de cara al Mundial de EEUU de 1994 (Díez, 2014), comenzando con la prohibición al portero de recoger los pases con la mano y la tarjeta roja para las faltas realizadas por el último defensa en 1990.

Todo ello permite ver que es en este momento histórico en el que se manifiesta la voluntad política y deportiva de reducir la violencia en el fútbol. Por ello, en el contexto de una investigación más amplia sobre el cambio en las identidades y competencias de los futbolistas de la época, se tomó la decisión de estudiar su relación con la violencia y las acciones antirreglamentarias. El objetivo por tanto es conocer cómo integraban la violencia dentro de sus identidades los futbolistas de los años ochenta y qué cambios se produjeron ante la presión reglamentaria de los años noventa para limitarla, de forma que aumente así el conocimiento de la cultura profesional de los futbolistas, pero también de la forma en que la violencia se relaciona con la masculinidad en la esfera deportiva. 
García-Martí, C.; Durán-González, J., y Gómez-López, M. (2017). El control de la violencia deportiva en el fútbol profesional español (1985-1995). RICYDE. Revista internacional de ciencias del deporte. 50(13), 322-338. https://doi.org/10.5232/ricyde2017.05001

\section{Método}

Teniendo en cuenta que esta investigación pretendía profundizar en las identidades y los discursos subjetivos de los futbolistas y entrenadores, se optó por desarrollar una metodología cualitativa, pues como señala Ortí (1996), la investigación cualitativa se ocupa de la comunicación simbólica que se estructura a través del lenguaje como sistema de signos y que al mismo tiempo es utilizada por los sujetos para expresar su subjetividad, transformándola en habla, en producción concreta, y es por tanto la técnica más adecuada cuando se pretende conocer los motivos y deseos profundos, la interpretación motivacional de los individuos y su imbricación en el universo estructural de los discursos, la dimensión semántica.

Dentro del paradigma cualitativo, se optó por utilizar las entrevistas en profundidad como técnica de investigación, junto con un análisis documental de fuentes secundarias, en este caso la prensa deportiva y generalista de la época. La entrevista en profundidad se caracteriza para Alonso (1998) por su potencialidad expresiva, para registrar la opinión, los valores y la experiencia vivida, y especialmente de sus prácticas a través del elemento biográfico, lo que permite contextualizar las ideas en un comportamiento, permitiendo así interpretar el discurso ideológico en su relación dialéctica con su práctica, observar sus límites o capacidad de imposición, y las tensiones que en ese quehacer acompañan a los sujetos y las contradicciones que implican. La entrevista en profundidad es fundamentalmente una narración abierta y en diálogo, por lo que se diseñó un guion temático que debía servir de guía y al mismo tiempo era modificado y ampliado durante el transcurso de la misma.

Y es que la investigación cualitativa se fundamenta en una cierta flexibilidad en su diseño, pues parte de la idea de que los datos que van emergiendo pueden hacer variar la noción que se tenía del objeto y del mejor modo de aproximarse a él. Por tanto, una investigación cualitativa es un proceso en marcha en el que confluyen temporalmente las distintas fases tradicionales del proyecto: el diseño metodológico, la recogida de datos y el análisis. Ello implica que el diseño no es una estructura cerrada, sino que se continúa definiendo en todos los momentos de la investigación a través de decisiones de diseño (Janesick, 1994). Lo mismo puede afirmarse de la pregunta de investigación, que inicialmente debe ser suficientemente amplia para no cerrar el campo y permitir, a través de la investigación, delimitar los auténticos campos de interés (Corbin y Strauss, 1990).

Dentro de ese diseño se realizó un muestreo teórico de conveniencia (Valles, 2000) que buscaba alcanzar la máxima variabilidad, limitada únicamente por la accesibilidad. Para ello se incluyeron la mayor variedad posible de perfiles profesionales, incluyendo a árbitros, periodistas y expertos junto a futbolistas y técnicos, aumentando así la capacidad de triangulación de los textos. También se incluyó la edad como variable de selección, junto con la posición en el campo y una variedad de recorridos profesionales y competitivos. No se trataba de lograr un entrevistado para cada perfil posible, sino de garantizar la heterogeneidad de itinerarios vitales para obtener la mayor variación en el discurso y por tanto garantizar la saturación discursiva (Alonso y Callejo, 1999), es decir, saturación del corpus, cuando todas las diferencias ya se han manifestado, y saturación de sentido, cuando no surgen nuevas posiciones ideológicas.

Debido a las dificultades propias de acceso al colectivo, muchos de ellos con un alto perfil mediático y profesional y problemas de agenda, los participantes se captaron a través de una técnica de bola de nieve, por la cual se solicitaba a cada participante que ofreciese un posible nuevo contacto. Así, se realizaron un total de 31 entrevistas, de los cuales 13 estaban activos como futbolistas en el periodo estudiado (1980-1995), 10 eran entrenadores en ese momento, 3 ejercían como árbitros, 3 desarrollaban una labor periodística, 1 era preparador físico y 1 más 
García-Martí, C.; Durán-González, J., y Gómez-López, M. (2017). El control de la violencia deportiva en el fútbol profesional español (1985-1995). RICYDE. Revista internacional de ciencias del deporte. 50(13), 322-338. https://doi.org/10.5232/ricyde2017.05001

era historiador. Las categorías resultaron en cualquier caso porosas, con 2 futbolistas convertidos en periodistas y 8 de ellos en entrenadores.

\begin{tabular}{|c|c|c|c|c|c|}
\hline Clave & $\begin{array}{l}\text { Año } \\
\text { nacimiento }\end{array}$ & $\begin{array}{l}\text { Nivel } \\
\text { futbolista }\end{array}$ & $\begin{array}{l}\text { Años } \\
\text { futbolista }\end{array}$ & $\begin{array}{l}\text { Nivel } \\
\text { Entrenador }\end{array}$ & $\begin{array}{l}\text { Años } \\
\text { entrenador }\end{array}$ \\
\hline Entrenador 1 & 1943 & 1a División & $1965-1978$ & 1a División & $1979-2004$ \\
\hline Entrenador 2 & 1950 & 1a División & $1973-1982$ & 1a División & 1985-???? \\
\hline Entrenador 3 & 1943 & 1a División & $1962-1974$ & 1a División & $1980-2008$ \\
\hline Entrenador 4 & 1957 & 1a División & $1976-1991$ & 1a División & 1995-???? \\
\hline Entrenador 5 & 1940 & Amateur & -- & 1a División & 1971-1988 \\
\hline Entrenador 6 & 1940 & 1a División & $1958-1971$ & 1a División & 1974-1998 \\
\hline Entrenador 7 & 1948 & 1a División & $1967-1980$ & 1a División & $1985-2008$ \\
\hline Entrenador 8 & 1946 & Amateur & -- & 2a División & 1990-1995 \\
\hline Entrenador 9 & 1946 & Amateur & -- & 1ạ División & 1985-???? \\
\hline Entrenador 10 & 1948 & 1ạ División & $1967-1984$ & 1a División & 1985-???? \\
\hline Portero 1 & 1970 & 1a División & $1990-2007$ & & \\
\hline Defensa 1 & 1962 & 1a División & 1981-1995 & & \\
\hline Defensa 2 & 1966 & 1ạ División & $1984-2002$ & & \\
\hline Defensa 3 & 1974 & 1a División & $1993-2004$ & & \\
\hline Defensa 4 & 1966 & 1a División & $1986-2005$ & & \\
\hline Defensa 5 & 1968 & 1ạ División & $1988-2003$ & & \\
\hline Defensa 6 & 1964 & 1ạ División & $1982-2000$ & & \\
\hline Defensa 7 & 1971 & 1ạ División & $1989-2004$ & & \\
\hline Centrocampista 1 & 1957 & 2a División & 1978-1992 & & \\
\hline Centrocampista 2 & 1967 & 1ạ División & $1984-2000$ & & \\
\hline Delantero 1 & 1965 & 1ạ División & $1982-1997$ & & \\
\hline Delantero 2 & 1963 & 1ạ División & 1981-1995 & & \\
\hline Delantero 3 & 1958 & 1a División & 1975-1989 & & \\
\hline
\end{tabular}

Figura 1: Características de la submuestra de profesionales del fútbol: entrenadores y futbolistas 
García-Martí, C.; Durán-González, J., y Gómez-López, M. (2017). El control de la violencia deportiva en el fútbol profesional español (1985-1995). RICYDE. Revista internacional de ciencias del deporte. 50(13), 322-338. https://doi.org/10.5232/ricyde2017.05001

De entre los catorce futbolistas, siete eran defensas, cinco de los cuales actuaban en el centro de la defensa, uno de entre ellos como líbero, y dos laterales. Además, había un centrocampista defensivo, un medio punta y un centrocampista de banda. Junto con ellos se encontraban tres delanteros y un portero. Salvo uno de ellos, que únicamente alcanzó la Segunda División, el resto compitió en Primera División. Los diez entrenadores habían sido futbolistas, con mayor o menor desempeño: seis habían jugado en Primera División, uno en Segunda División, y tres en categorías inferiores, dos de ellos retirados jóvenes por lesiones. De los tres árbitros, dos arbitraban en Primera División en el periodo analizado y uno lo hacía en categorías inferiores.

Tras realizar el contacto y explicar el motivo de la investigación, se procedía a concertar una cita para la realización de la entrevista. Se privilegió la realización en un espacio neutro, como un espacio público, pero en ciertos casos se tuvo que recurrir a los lugares de trabajo de los entrevistados, por motivos de agenda. Para ampliar las posibilidades de captación se recurrió a participantes fuera del ámbito geográfico del entrevistador, lo que obligó a realizar las entrevistas por teléfono. Finalmente, se llevaron a cabo 20 entrevistas presenciales, 9 telefónicas y 2 por videoconferencia.

El entrevistador mantuvo un cuaderno de campo y diario de investigación, en el que realizaba informes de cada entrevista en las que reflejaba la información pertinente sobre el momento de la entrevista, el lugar, los posibles elementos externos influyentes, la comunicación no verbal de los participantes y las nociones teóricas o analíticas surgidas espontáneamente, para contextualizar y enriquecer el análisis posterior.

Las entrevistas tuvieron una duración de entre 30 y 120 minutos, dependiendo de la disponibilidad y del contexto. Todas fueron recogidas con una grabadora y transcritas por el equipo investigador para su posterior análisis. El análisis fue realizado con la ayuda del programa informático de análisis de textos Atlas.ti 6.

Este análisis comenzó con un análisis puramente semántico, que Ortí (1996) denomina intratextual, segmentando el conjunto de datos en segmentos significativos que presentasen una coherencia interna, categorizándolos y codificándolos temáticamente y en función también de los perfiles de los entrevistados, del tipo de lenguaje y del momento de aparición, todo ello en un proceso dialéctico por el que los nuevos hallazgos daban lugar a nuevas categorías y viceversa. Ese análisis se complementaba con el que Orti (1996) denomina transtextual, la apertura del texto a la realidad social amplia y a la teorización, o siguiendo a Alonso (1998), la introducción del texto en su contexto semiótico, social, histórico y cultural, de forma que los discursos recogidos sean integrados en los grupos sociales de referencia y en el contexto histórico en el que se producen.

La cuestión de la validez de las técnicas cualitativas es conflictiva. Para Alonso (1998), la entrevista en profundidad involucra necesariamente la subjetividad del investigador, que por tanto crea el objeto y no simplemente lo descubre, por lo que las nociones de replicabilidad o falsabilidad están fuera de lugar. Para Ortí (1996) no se trataría de una limitación de la investigación cualitativa sino de una propia de una sociología inmersa en la "inevitabilidad epistemológica de la interpretación" (1996, p. 207).

Desde la tradición anglosajona sí se han considerado necesarios criterios de calidad propios para la investigación cualitativa (Corbin y Strauss, 1990) siendo una de las propuestas más aceptadas la de Erlandson y colegas (Erlandson, Harris, Skipper, y Allen, 1993), que determina tres criterios para garantizar la validez y fiabilidad de los datos: credibilidad, o hasta qué punto son representativos los datos; transferibilidad, o hasta qué punto la muestra es representativa; 
y dependibilidad, la posibilidad de investigadores externos de confirmar los hallazgos. En nuestro caso, la credibilidad estaría garantizada tanto por la triangulación de técnicas, en concreto cuadernos de campo, entrevistas en profundidad y análisis documental, como por la participación de varios investigadores; la transferibilidad lo estaría por el muestreo teórico y la saturación; y la dependibilidad, lo sería por la exposición pública de los resultados y la transparencia en la descripción del diseño metodológico y el análisis.

\section{Resultados}

El análisis de las entrevistas nos ha permitido comprender que el uso de la violencia y de otras estrategias antirreglamentarias o contrarias al espíritu del juego - fair play - formaban parte de una cultura compartida por todos los actores del fútbol profesional, tanto futbolistas como entrenadores, árbitros, directivos, público y periodistas. A lo largo del tiempo se había creado un consenso social sobre las conductas y actitudes esperables por parte de sus miembros, sobre lo que era legítimo hacer y lo que no lo era, que funcionaba como un marco moral normativo. Este marco trascendía la norma escrita, en este caso el reglamento, pues por un lado regulaba actuaciones que no estaban contempladas en él, y por el otro se situaba en una posición de preeminencia frente a la norma escrita, es decir, prevalecía en caso de contradicción entre ambas.

Si debiésemos resumir en un concepto cuál era, a ojos de nuestros entrevistados, el consenso en los años previos a la zona, diríamos que se consideraba que todo era fútbol. Ello incluía no solo el desarrollo del juego durante el partido, sino que todo lo que sucedía antes, durante y después y todos los actores implicados. Así, era legítimo todo aquello que el club como institución pudiera hacer, como reducir las dimensiones del campo pintando las líneas o "destrozar" o "enfangar los terrenos de juego para imposibilitar el juego del rival" (Periodista 1). Estas eran decisiones del club y del entrenador, que no distinguían entre medios internos y externos, entre medios legítimos e ilegítimos de lograr la victoria. Esta victoria era el único objetivo de la institución, del equipo y también del público, que tenía un rol fundamental en su consecución apoyando y animando, pero sobre creando un ambiente de intimidación y violencia "masiva (...) almohadillas, botes, el árbitro saliendo protegido por los escudos policiales... eso lo he vivido yo" (Árbitro 1). Una violencia no minoritaria, sino practicada por el conjunto del estadio y que se retroalimentaba con las acciones de los futbolistas, especialmente los defensas más duros, "el futbolista parte piernas, que casi llevaba medallas, o las muescas en el Colt" (Árbitro 1) que eran "los paladines e ídolos de la afición" (Periodista 1) dentro de un fútbol "muy castrense, muy blanco y negro, muy esforzado, muy de macho de pelo en pecho y tal, de anuncio de coñac y de puros" (Periodista 2).

Todo ello derivaba en un criterio arbitral distinto para locales y visitantes que hacía que "jugar fuera de casa era una temeridad entonces" por esa intención declarada del equipo local de “intimidación del rival, de intentar achicarlo moralmente" (Delantero 1)

Claro, cuando ibas fuera, te hacían falta y la gente aplaudía. Entonces claro, el otro, la próxima vez más fuerte. Y el árbitro miraba para otro lado, le advertía, pero le advertía... (Delantero 2)

Se trataba de una intimidación en primer lugar psicológica, muchas veces mediante faltas que no buscaban un beneficio inmediato, sino avisar condicionar el comportamiento del contrario a lo largo del partido, "para avisarte, oye tú, el partido no va a ser fácil" (Delantero 2) y más allá: 
García-Martí, C.; Durán-González, J., y Gómez-López, M. (2017). El control de la violencia deportiva en el fútbol profesional español (1985-1995). RICYDE. Revista internacional de ciencias del deporte. 50(13), 322-338. https://doi.org/10.5232/ricyde2017.05001

Pero era un hábito, pegaban por pegar, porque así, era una forma de marcar el territorio, para que no te olvides de mí para otras veces que nos encontremos y cosas así. (Centrocampista 1)

Parte de esa estrategia psicológica era la provocación, el intercambio verbal y cualquier método de desestabilización que se le pudiese ocurrir al jugador. El marcaje individual provocaba que dos jugadores se mantuviesen juntos durante todo el encuentro, y en ese duelo que se establecía, "había partidos en los que era más el verbal que el táctico (...) el me pico tú me picas, a ver quién cae más rápido" (Defensa 1). Guerras psicológicas que, al igual que la violencia, podían detenerse al finalizar el partido o dar lugar a enemistades prolongadas, incluyendo "ofertas de venganza" (Árbitro 1). Esos choques podían ser el fruto espontáneo del enfrentamiento deportivo, pero en según qué casos caían también dentro de las competencias de los entrenadores, que estudiaban a los contrarios y sugerían al jugador "pícalo para que lo echen" (Defensa 2). Esa pelea psicológica se desarrollaba fuera del radar del árbitro, que no tenía medios para controlar todo lo que sucedía en el campo:

Yo creo que esa violencia de la que hablábamos entonces era, pues como todo, es decir, era oculta, y entonces como era oculta, salvo que el árbitro estuviera especialmente diligente, y tuviera, pues oiga, mire usted bien que fíjese lo que me hace éste, y tú dieras crédito a lo que te acababan de decir, pasaba completamente desapercibido. (Árbitro 1)

Y es que existían multitud de ángulos muertos en el trabajo arbitral, alejados de su control, que alcanzaba su cénit en esas acciones a balón parado en las que "te agarraban, te pegaban, nadie se enteraba, ¿iquién se iba a enterar!? (Delantero 1). Ese clima de violencia tenía su corolario en acciones de extrema dureza que podían considerarse agresiones. Esas acciones tenían, cotidianamente, consecuencias muy graves para la salud de los jugadores, hasta el punto de poner en peligro sus carreras deportivas, y sin embargo apenas eran castigadas por la "permisividad arbitral" (Árbitro 1):

Durísimo. Pero durísimo. Hombre, yo tengo dos fracturas de tibia, de dos patadas. (...) Y un bestia, por no llamarle otra cosa más dura, me pegó una patada por detrás que me agarró... el menisco liquidado. (...) Bueno, al que... uno de los dos, que me partió la pierna en [estadio local], no se me olvida, ese chico no le sacaron ni tarjeta. (Entrenador 1)

Todo ello formaba parte de una ética profesional, puramente instrumental, que se situaba por encima del reglamento y del marco moral del fair play, en un contexto como hemos visto en el que la autoridad legal carecía de legitimidad por su permisividad y limitaciones:

Y yo tenía claro que si yo les tenía que meter una patada o lo que fuera pues es que era parte de mi trabajo. (...) Pero no quiero decir con esto que estoy de acuerdo con que se den patadas, pero es parte del fútbol. (Defensa 3)

Existe pues la idea de que la violencia forma parte del fútbol y de la profesión, que es una herramienta legítima. Al mismo tiempo, hemos visto que esa violencia podía tener consecuencias graves para los futbolistas, en forma de lesiones. Los futbolistas no son ajenos a esta realidad, por lo que en aquella época debían mantenerse en una ambigüedad moral, en una suspensión del juicio ético:

Sí, pero por lo que hablábamos, los centrales antes eran centrales. Tú vas a coger a este delantero y lo vas a secar, este no se mueve. Tu función es esta, tú no sabes jugar el balón, tú no sabes nada. Y luego porque tampoco 
teníamos dos dedos de cabeza. ¿Cuántos jugadores se han retirado por lesiones porque has ido a un, en un momento malo, y has entrado con todo, sabiendo qué si lo quitas de en medio es un jugador menos, y si encima es importante? Lo que pasa es que si a un jugador lo tienes para esas cosas, pero no creo que nadie... (Defensa 1)

No solo el jugador debía actuar sin dos dedos de cabeza para evitar asumir las consecuencias de su acción, sino que ésta remite a una cultura ocupacional y una autoridad que le tiene para esas cosas, propiciando un comportamiento peligroso sin que el entrenador lo pida ni que el jugador lo haga conscientemente.

A pesar de esas consecuencias dramáticas para la carrera profesional de algunos, la violencia estaba legitimada tanto entre defensas como entre delanteros, pues estos últimos entendían "que ellos hacían su labor, igual que teníamos nuestros defensas" y que "el arbitraje era el que era" (Delantero 2). Es por ello que "antes pues bueno, en el campo se podían hacer muchas cosas, y el tópico que se dice, que todo quedaba en el campo" (Centrocampista 2), y al acabar el partido "nos dábamos la mano y sabíamos que era parte de este deporte" (Defensa 3).

La referencia ética superior era pues la ética profesional, que entendía que cada uno estaba legitimado para hacer todo aquello que considerase necesario, pues el contrario era "el enemigo, un tipo que está robándome a mí mi reputación, la comida de mi familia, la letra de mi casa. Tú eres un estorbo en mi vida" (Delantero 3). Es decir, que la identidad de los futbolistas estaba marcada por una masculinidad agresiva y por una autoestima fundamentada en su rol productivo como trabajadores y reproductivo como cabezas de familia y proveedores, típico de la modernidad.

Esta cultura futbolística y profesional estaba asociada en último término a la furia española, el supuesto estilo característico del fútbol español basado en la agresividad, el sacrificio y la voluntad de victoria, un término nacido con la medalla de plata de los Juegos Olímpicos de Amberes de 1920 (Simón Sanjurjo, 2014) y posteriormente utilizado con fines propagandísticos por el régimen franquista (Davila, 1972; García Candau, 1980). El final de los años ochenta va a ver transformada esta idea bajo la influencia de dos elementos inéditos: la generalización de las retransmisiones televisivas con muchos más medios técnicos que hasta entonces y los cambios en el criterio arbitral. En el origen del proceso estaba la pérdida de la legitimidad que la violencia mantenía en la práctica del fútbol profesional. Se trata de un fenómeno global que engarza con el cambio del fútbol competición al fútbol espectáculo, pero también con el trauma que supusieron las tragedias en los estadios de Heysel y Hilborough, y en España la de Sarrià, que llevó a que en unos años esos lanzamientos de piedras y almohadillas quedasen "desterrados" (Árbitro 1) permitiendo a los árbitros desarrollar su labor sin presiones.

El impacto de la televisión es destacado por todos los entrevistados. Con la apertura del mercado televisivo, y especialmente con la llegada de Canal + , se multiplicó el número de partidos retransmitidos, pero sobre todo el número de cámaras instaladas, que aumentaron exponencialmente la capacidad de captar todo aquello que hasta ese momento pasaba desapercibido, y en primer lugar la violencia.

Cuando vino Canal+, por ejemplo, que era una cadena que te metía diez cámaras en el campo, y claro, en esa época se hacían cosas que ahora serían impensables, no serían impensables porque se siguen haciendo, pero ahora se ven. (Defensa 3) 
García-Martí, C.; Durán-González, J., y Gómez-López, M. (2017). El control de la violencia deportiva en el fútbol profesional español (1985-1995). RICYDE. Revista internacional de ciencias del deporte. 50(13), 322-338. https://doi.org/10.5232/ricyde2017.05001

La identificación de la violencia no era únicamente resultado de una capacidad tecnológica, sino de una postura ética de rechazo a la violencia que se integraba en la voluntad de crear un producto más atractivo y espectacular:

Nosotros desde muy primera hora empezamos a apostar porque se respetara a quien aportaba creatividad y calidad al juego, porque entendíamos que eso era lo que le iba a hacer evolucionar. Si al final el matón de 34 lleno de cicatrices, conseguía que el chaval de 18 no jugara en todo el partido porque salía asustado desde el túnel de vestuarios, pues nosotros intentábamos combatir eso (...) porque entendíamos el fútbol de otra manera mucho más moderna, y que tenía que ver más con el espectáculo más que con otra cosa. (Periodista 1)

Para el equipo de Canal + la violencia era éticamente reprobable, y mala para la industria del fútbol. El espectáculo era imposible sin estrellas, sin goles y sin juego. El fútbol profesional debía deshacerse de la violencia si quería evolucionar y abrirse a nuevos estratos de la sociedad. Ampliar el mercado del fútbol formaba parte de la lógica empresarial de las televisiones, que tras gastar miles de millones necesitaban rentabilizar sus inversiones atrayendo a la mayor cantidad posible de público. Pero para los periodistas esa lógica económica se entremezclaba de forma indistinguible con la búsqueda de la renovación del fútbol español. Y la denuncia era mucho más efectiva si se apoyaba en imágenes, porque "nadie soporta ver esa patada repetida siete veces" (Periodista 3).

Esa visibilidad de la violencia generó su problematización dentro de la propia comunidad futbolística, que empezó a constatar que la violencia ya no podía considerarse como expresión adecuada de la masculinidad.

Es decir, la nobleza, la fuerza, eso yo creo que estaba, estuvo y estará bien visto, pero claro, cuando ya se exceden los límites, y sobre todo cuando cómo te digo lo que determina muchísimo es la lesión, y si es la lesión grave de un jugador, eso es lo que realmente determina el cambio y la reacción de esto ya no puede seguir por este camino, hay que proteger al jugador. (Árbitro 2)

Es la presión de "los espectadores, la prensa, los medios de comunicación" (Árbitro 2) que fuerzan al gobierno del fútbol a cambiar. Surge así un discurso nuevo que considera que "el fútbol es un espectáculo en el que no solo se tiene que proteger a los grandes futbolistas, sino que hay que proteger al futbolista como espectáculo" (Defensa 4). Los futbolistas crean un espectáculo, y el público acude a ver ese espectáculo, lo que constituye un cambio radical frente a la concepción del fútbol como enfrentamiento y al público como seguidor interesado únicamente en la victoria. Ese cambio genera resistencias, porque supone degradar prácticas que hasta entonces eran legítimas y "que también son fútbol" (Centrocampista 2), parte de la identidad de varias generaciones, y en segundo lugar porque supone desclasar a una parte de los futbolistas que también son parte de la profesión, en especial los defensas:

Es que ahora se promulga lo de es que a este hay que cuidarle, a Messi hay que cuidarle, a Neymar hay que cuidarle, ya, pero también hay que cuidarle al central del Elche, al lateral del Murcia, ¿sabes? Creo que todos tenemos derecho, no solo a las grandes estrellas, porque llevan el balón y son más vistosos y son los que dan un plus a la Liga porque aquí somos todos jugadores. (...) Por eso te digo que todos los jugadores debemos ser iguales y que esto es parte del fútbol, y si no quieres que te den patadas... 
García-Martí, C.; Durán-González, J., y Gómez-López, M. (2017). El control de la violencia deportiva en el fútbol profesional español (1985-1995). RICYDE. Revista internacional de ciencias del deporte. 50(13), 322-338. https://doi.org/10.5232/ricyde2017.05001

yo siempre digo a mis jugadores, si no quieres que te den patadas sueltas el balón antes, porque las patadas son parte de este deporte (Defensa 3).

Se trató sin embargo de un cambio global que impuso su hegemonía, como vemos en el carácter defensivo, o impugnador, de estos discursos de resistencia, pues estaba apoyado por los medios de comunicación y fue impulsado por las instituciones que gobiernan el fútbol, como la FIFA, que introdujo cambios normativos como la tarjeta roja a las faltas del último defensor y que a través de las federaciones y el estamento arbitral introdujo un cambio en la interpretación del reglamento que es cambio de cultura, producido por "esa presión que el propio fútbol ha generado de protección al jugador" que provoca "la clarificación de qué debe ser y qué no" (Árbitro 1), es decir, el aumento del rigor disciplinario.

Así, vemos en el gráfico, tras unos primeros años de rodaje, entre la temporada 76/77 y la 82/83 las tarjetas amarillas estaban estabilizadas alrededor de las dos por partido (máximo de 2.28, mínimo de 1.92). En los años siguientes aumentan las tarjetas amarillas, que llegan a alcanzar las 2.93 por partido en la temporada $85 / 86$, para volver a bajar hasta las 2.53 en la $87 / 88$. A partir de ahí, se disparan hasta alcanzar un pico de 5.24 tarjetas amarillas en la temporada 95/96. Por su parte, las tarjetas rojas se mantienen entre 0,1 y 0,2 por partido hasta la temporada 89/90 (0.22), para doblarse en los años siguientes hasta alcanzar también un pico en la 95/96 (0.45).

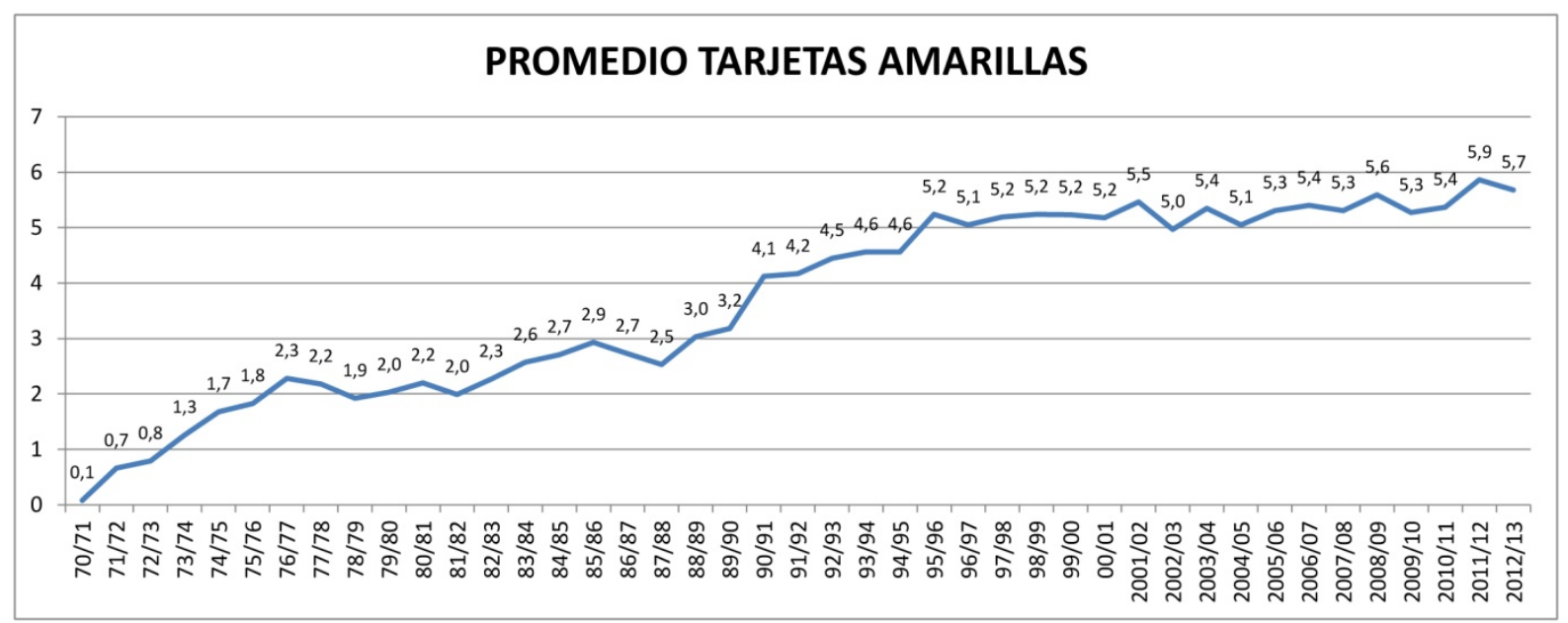

Figura 2: Promedio de tarjetas amarillas. Fuente: elaboración propia con datos de BDFútbol.com

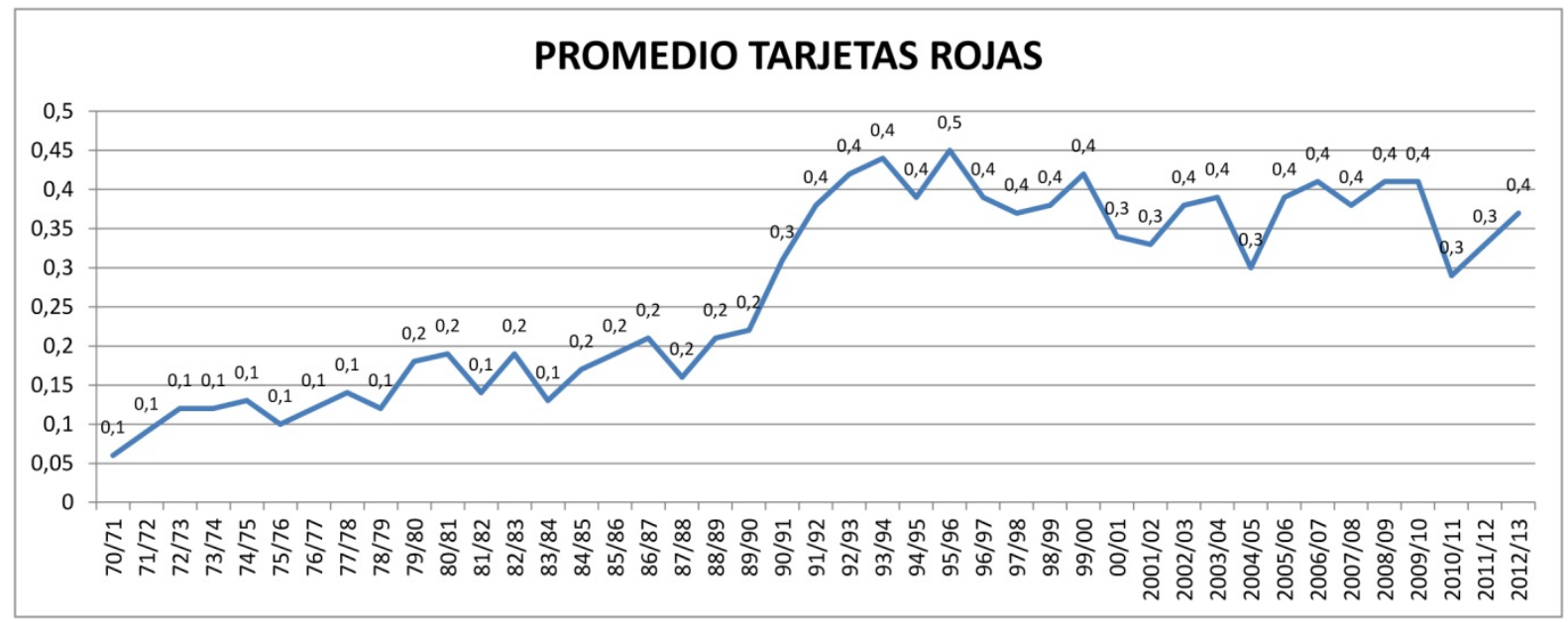

Figura 3: promedio de tarjetas rojas. Fuente: elaboración propia con datos de BDFútbol.com 
Este aumento de las tarjetas no es el resultado de cambios reglamentarios, que se limitan a la tarjeta roja para el último defensor, por lo que deben atribuirse a los cambios en la interpretación del reglamento que "emana" de la federación y que por ejemplo durante "un tiempo insistió mucho en las entradas", hasta convertir las faltas por detrás "en anecdóticas, y antes se veían cada dos por tres" (Árbitro 3). Se trata de un aumento general del rigor en la aplicación del reglamento, del fin del clima de impunidad que permitía las faltas reiteradas, los avisos, la violencia subrepticia y la violencia desproporcionada, dando paso a uno en el que "el defensa tiene que tener mucho cuidado, porque sabe que a la mínima le pueden expulsar" (Delantero 2). En consecuencia, surge en primer lugar la exigencia de control emocional para los defensas, que "van todos fuertes, pero bueno, se miden más" (Defensa 2).

Además, la exposición mediática ha modificado las ventajas de la violencia subrepticia y de las guerras psicológicas, no solo porque una vez expuestas estas acciones pueden ser revisadas de oficio, sino porque pasan a formar parte de la reputación del jugador, tanto para los árbitros, "que ya te van cogiendo" (Defensa 2), como por parte de la opinión pública, porque "cualquier jugador que cometa, yo que sé, que cometiera falta alevosa quedaría denunciado en el mundo entero" (Delantero 1). En consecuencia, el juego subterráneo "se tiene que dejar de hacer aunque... se hace lo que se puede" (Portero 1).

Y es que, en principio, todo ello no significa un cambio ético, "al jugador no le ha hecho mejor persona que hace 30 años" (Árbitro 1), simplemente tienen que andarse "con pies de plomo en hacer acciones que antes las podías hacer y ya no" (Defensa 2). Los defensas habrían adaptado su práctica a la nueva relación coste/beneficio, sin abandonar la ética instrumental, y seguirían actuando "de la misma forma" (Defensa 5).

Sin embargo, este último añade que ahora "cualquier acción más bruta se castiga más que antes", y el Defensa 2 dice que antes había entradas "fuertes, que si las ves ahora serían todas de tarjeta roja", y el Defensa 3 que en los años setenta "se hacía de todo" como llevar alfileres en las medias, y que muchas de esas cosas han pasado "a la historia". Es decir, que implícitamente todos reconocen que existe un límite en la violencia aceptable, que no todo es puro cálculo instrumental. Para ellos mismos, aunque se muestren comprensivos con sus compañeros, muchas de las acciones que eran comunes en los años setenta les provocan un rechazo moral, se sitúan por debajo de sus estándares. Sin darse cuenta, estos estándares han ido subiendo en el conjunto de la sociedad y del fútbol profesional y ese proceso también les ha afectado.

De alguna forma, la ética profesional es en realidad un conjunto vacío que solo toma significado cuando se analiza lo que cada grupo en cada momento considera que la constituye y lo que no. Bajo la supuesta preeminencia de la lógica instrumental se esconde que la consideración de los alfileres, de los agarrones en el área, de los escupitajos o de las entradas por detrás cambia con el tiempo, y que la tendencia de las últimas décadas es hacia la expulsión de cada vez mayores instrumentos del ámbito de lo aceptable. El límite es por lo tanto social, construido por la comunidad, y evoluciona con el tiempo. Por tanto, lo que sucedió en España es que el equilibrio de poder entre los actores varió en favor de los defensores de la protección del futbolista gracias al apoyo de los medios y las instituciones, dentro de ese discurso que hemos visto del fútbol espectáculo ligado a la transformación del negocio.

Ese cambio no solo afectó a los discursos, sino también a las prácticas. La reducción de la violencia modificó las condiciones de trabajo de los defensas, desprovistos de su principal herramienta de trabajo, y los obligó a desarrollar nuevas cualidades y habilidades, generando una transformación de su identidad y cultura profesional. Así, si un defensa de los setenta y ochenta es loado por su compañero por no ser "un dechado de técnica, pero de fuerza, de 
García-Martí, C.; Durán-González, J., y Gómez-López, M. (2017). El control de la violencia deportiva en el fútbol profesional español (1985-1995). RICYDE. Revista internacional de ciencias del deporte. 50(13), 322-338. https://doi.org/10.5232/ricyde2017.05001

valentía, de salto, de correr, era un fenómeno" (Entrenador 1), un defensa de los noventa considera que lo que hace a los defensas de talla mundial es "que sean buenos tácticamente, que sepan elegir el espacio, la zona que tienen que ocupar, y sobre todo decidir cuál es el momento de acometer el hombre a hombre con el jugador, de saber cuándo tienen que dejarlo, de saber cuándo tienen que ocupar un espacio..." (Defensa 5). Un perfil con un fuerte contenido cognitivo y muy alejado de la masculinidad tradicional y agresiva y de las proezas físicas que animaban las descripciones de los defensas en los años setenta y ochenta.

\section{Discusión}

La literatura previa muestra dos interpretaciones de la violencia deportiva interna: la Escuela de Leicester sostiene que el deporte es fundamentalmente juego pacificado y por tanto muestra del proceso de civilización, siendo la violencia interna puramente instrumental, y por tanto racional y limitada. Por su parte, los estudios de género sostienen que la violencia deportiva es parte de una masculinidad agresiva que es hegemónica en el fútbol, muestras de la cual han sido identificadas en los estudios ocupacionales sobre la profesión de futbolista. ¿Es por tanto el fútbol un espacio de civilización o de exaltación de la masculinidad agresiva? Entendemos que nuestros resultados ofrecen la posibilidad de un diálogo entre ambas tradiciones que permite que se complementen, integrando la evidencia de un proceso de pacificación y también de una cultura masculina tradicional en el deporte.

En primer lugar, hemos podido comprobar que la violencia de los futbolistas no era únicamente instrumental y ejercida de forma aséptica por los futbolistas, como suponía Dunning (1986) de unos profesionales. Por el contrario, esa mezcla de guerra psicológica, violencia subrepticia y faltas alevosas se llevaba a cabo en un ambiente altamente emocional y formaba parte de una masculinidad orgullosa y tradicional fundamentada en la noción del futbolista como trabajador y cabeza de familia. Era esta doble condición la que le otorgaba la legitimidad para saltarse la norma y la ética deportiva, pues por encima de ambas estaba su derecho al cuidado de su familia y la relación directa entre su autoestima y esta capacidad de proveer. A través de esta noción compartida de masculinidad tenemos una violencia que es al mismo tiempo instrumental, pues busca un beneficio, y expresiva, pues expresa una identidad.

Parecería por tanto que la idea de los estudios de género sobre el dominio de una masculinidad hegemónica en el fútbol sería correcta, y este sería un espacio de reproducción de los valores masculinos más tradicionales. Sin embargo, hemos visto que se produjo un cambio en el discurso, con la llamada al espectáculo y a la protección del jugador, que modificó realmente el criterio arbitral y la violencia ejercida en el campo, que se redujo sensiblemente. ¿Cómo entender este cambio? ¿Como ejemplo paradigmático de la curva civilizatoria de Elías, como un fenómeno de feminización de la cultura deportiva, o como efecto colateral de la mercantilización del fútbol?

En apoyo de la primera hipótesis tenemos ese disgusto físico típicamente civilizatorio frente a la violencia más excesiva que encontramos en las nuevas generaciones, tal y como esperaría Elías (1992) de aquellos que no han estado en contacto con la violencia pasada. En la construcción teórica de Elías, el proceso civilizatorio conlleva la reducción de la violencia expresiva por culpa de dos fenómenos: el aumento del poder coercitivo del Estado y la extensión de las cadenas de interdependencia. A medida que dependemos más los unos de los otros y de que el estado tiene mayor capacidad de controlar, se reduce la violencia. En nuestro caso, el poder central, en este caso las organizaciones futbolísticas, ya tenían capacidad para sancionar la violencia y únicamente se deciden a aplicarlo por la presión de la opinión pública y por la propia transformación del fútbol en producto mediático, es decir, su mayor dependencia de los medios de comunicación, que a su vez modulan la opinión pública. Es decir, 
que la reducción de la violencia es una exigencia de las televisiones en su afán por hacer el producto atractivo a nuevas capas sociales, y solo en ese sentido podría entenderse como el producto de un aumento de las cadenas de interdependencia. Entre esas nuevas capas está el público femenino, sin que sin embargo este haya sido protagonista del cambio ni lo haya liderado de forma consciente u organizada, por lo que la posible feminización del fútbol ha sido únicamente resultado de los intereses de los medios de comunicación, a su vez dominados mayoritariamente por hombres.

Independientemente del origen, está la cuestión de las consecuencias. ¿Esta limitación de la violencia modificó la cultura profesional de los futbolistas, y si así fue, qué consecuencias tiene para la noción de masculinidad hegemónica? Como hemos dicho, para los futbolistas, $\mathrm{y}$ especialmente para los defensas, resulta muy difícil, sino imposible, ejercer una crítica moral hacia sus predecesores, pues prima la solidaridad profesional y sobre todo la noción de continuidad de una comunidad. Se tiende pues a negar, a pesar de las evidencias, un cambio ético o cultural, y se reduce todo a un mayor control sancionador y mediático. Los defensas seguirían siendo los mismos, pues seguirían primando la ética profesional sobre el fair play.

Sin embargo, este discurso es en realidad un discurso de resistencia, pues así se articula en los discursos recogidos, frente a un discurso que se percibe hegemónico, como es el del fútbol espectáculo y la protección al futbolista. Esta noción convierte al futbolista fundamentalmente en artista, lo que le hace no solo merecedor de la protección arbitral, sino que traslada el origen de su autoestima y de su identidad, desde el eje de la producción y la reproducción - el padre de familia - al eje de la creatividad y la inteligencia. El futbolista no es un guerrero que se sacrifica para ofrecer una victoria a su comunidad, sino el responsable de un espectáculo que el público paga por presenciar.

El estudio de Llopis (2008) apunta a que este cambio de discurso no se ha generalizado en la práctica formativa ni entre el público de los estadios, pero nuestra investigación sí ha identificado que goza de una posición hegemónica entre los profesionales: no solo es mayoritaria, sino que quienes la rechazan lo hacen asumiendo su imperio. Que se haya producido una reducción de la violencia no implica que el fútbol profesional sostenga un discurso menos masculino, y como hemos visto los futbolistas rechazan que esta disminución haya supuesto un cambio en sus valores y su cultura profesional. Sin embargo, sí hemos identificado cambios como consecuencia de la menor violencia, como es la exigencia de mayor control emocional e inteligencia táctica, por lo que podemos afirmar que se producen cambios en la definición de la masculinidad hegemónica en el tiempo, y que estos cambios son el resultado de presiones sociales externas y de evoluciones internas de la institución, como en este caso el proceso de mercantilización. La masculinidad hegemónica no es única e invariable, sino que como sostiene Connell (Connell \& Messerschmidt, 2015) en su revisión del concepto, es un constructo dinámico y variable que responde a un estado de fuerzas, y no solo entre hombres y mujeres, sino entre grupos sociales e instituciones con distintos intereses y capitales simbólicos. Nos acercaríamos por tanto a la idea de Bourdieu (1997) de lo social como campo de fuerzas en el que los actores intentan imponer sus ideas para valorizar su capital y mejorar su posición. 


\section{Conclusiones}

Hemos identificado una reducción de la violencia en el fútbol español de principios de los años noventa, fruto de la mayor disciplina arbitral y mediática. Ello conllevó la pérdida de algunas de las herramientas habituales de los defensores, y la necesidad para estos de desarrollar nuevas habilidades y por tanto, una nueva fuente de autoestima en la construcción de su identidad. Ese proceso de pacificación sigue hasta cierto punto los patrones del proceso descrito por Elias, pero se diferencia en la ausencia de una distinción entre violencia instrumental y expresiva, y también en el origen del proceso, que parece estar en el cambio de modelo de negocio del fútbol. Todo ello se produce en una cultura profesional de una masculinidad tradicional, que sin embargo se ha visto modificada por la asunción del llamado deporte espectáculo. La masculinidad en el deporte es por tanto el resultado de un juego de fuerzas en permanente evolución, y que no tiene siempre necesariamente el dominio masculino como fuerza motora.

Este análisis de la violencia y su relación con la masculinidad y la identidad de los futbolistas profesionales nos permite ver la imbricación de estos en el fenómeno global de la industria deportiva, y cómo su evolución afecta a las prácticas y creencias internas del colectivo, al mismo tiempo que nos permite identificar los rasgos que constituyen su tradición y cultura compartida y que, aunque evolucionan, se mantienen estables en parte en una comunidad cerrada y artesanal como ésta.

Esta investigación se restringe a las vivencias de los profesionales, dejando fuera el estudio de la audiencia, es decir, del público, y los posibles cambios en su aprensión y comprensión del fútbol con el advenimiento del fútbol espectáculo. Tampoco se ha llevado a cabo un análisis de contenido de la producción periodística y mediática de la época para identificar ese cambio de discurso, aunque sí se han incluido entrevistas con periodistas. Estas limitaciones abren por tanto un campo para la investigación futura, que debería poder trazar una descripción completa del cambio del modelo de negocio del fútbol en España, de sus actores y de las consecuencias para estos, sus identidades y discursos.

\section{Referencias}

Alonso, L. E. (1998). La mirada cualitativa en sociología: una aproximación interpretativa. Madrid: Editorial Fundamentos.

Alonso, L. E., y Callejo, J. (1999). El análisis del discurso: del postmodernismo a las razones prácticas. Reis, $88,37-73$. https://doi.org/10.2307/40184203

Bertrand, J. (2009). Entre «passion» et incertitude: la socialisation au métier de footballeur professionnel. Sociologie du Travail, 51(3), 361-378. https://doi.org/10.1016/j.soctra.2009.06.002

Best, S. (2010). The Leicester School of Football Hooliganism: an evaluation. Soccer \& Society, 11(5), 573-587. https://doi.org/10.1080/14660970.2010.497352

Bonaut, J. (2010). El eterno problema del fútbol televisado en España: una perspectiva histórica de la lucha por los derechos de retransmisión de la Liga de Fútbol Profesional (LPF). Comunicación y sociedad, 23(2), 71-96.

Bourdieu, P. (1997). Razones prácticas. Sobre la teoría de la acción. Barcelona: Editorial Anagrama.

Carrigan, T.; Connell, B., \& Lee, J. (1985). Toward a New Sociology of Masculinity. Theory and Society, 14(5), 551-604. https://doi.org/10.1007/BF00160017 
García-Martí, C.; Durán-González, J., y Gómez-López, M. (2017). El control de la violencia deportiva en el fútbol profesional español (1985-1995). RICYDE. Revista internacional de ciencias del deporte. 50(13), 322-338. https://doi.org/10.5232/ricyde2017.05001

Connell, A. R. W., \& Messerschmidt, J. W. (2015). Hegemonic Masculinity: Rethinking the Concept. Gender and Society, 19(6), 829-859. https://doi.org/10.1177/0891243205278639

Corbin, J., \& Strauss, A. (1990). Basics of qualitative research: Grounded theory procedures and techniques. Londres: SAGE.

Davila, L. (1972). Política y deporte. Barcelona: Editorial Andorra.

Day, D. (2011). Craft coaching and the «Discerning Eye» of the coach. International Journal of Sports Science \& Coaching, 6(1), 179-195. https://doi.org/10.1260/1747-9541.6.1.179

Díez, Ó. (2014). Las reglas experimentales de los 90: el fútbol que estuvo a punto de ser. Cuadernos de Fútbol, (51).

Durán, J. (1996). El vandalismo en el fútbol. Una reflexión sobre la violencia en la sociedad moderna. Madrid: Gymnos.

Dunning, E.; Murphy, P., \& Williams, J. (1986). Spectator violence at football matches: towards a sociological explanation. The British Journal of Sociology, 37(2), 221-244. https://doi.org/10.2307/590355

Dunning, E., \& Sheard, K. (2005). Barbarians, gentlemen and players: A sociological study of the development of rugby football. Londres: Routledge.

Elias, N., \& Dunning, E. (1966). Dynamics of group sports with special reference to football. The British Journal of Sociology, 17(4), 388-402. https://doi.org/10.2307/589186

Elias, N., \& Dunning, E. (1992). Deporte y ocio en el proceso de civilización. México D.F.: Fondo de Cultura Económica.

Erlandson, D.; Harris, E.; Skipper, B., \& Allen, S. (1993). Doing naturalistic inquiry: A guide to methods. Newbury Park: SAGE.

García, C.; Gómez, M., y Durán, J. (2016). Los planes de saneamiento y la conversión de los clubes de fútbol profesionales en Sociedades Anónimas Deportivas. Materiales para la Historia del Deporte, 14, 1-18.

García Candau. J. (1980). El fútbol, sin ley. Madrid: Penthalon Ediciones.

Giulianotti, R. (2000). Football: a sociology of the global game. Cambridge: Polity Press.

Harding, J., \& Taylor, G. (2003). Living to Play: From Soccer Slaves to Socceratti. A Social History of the Professionals. London, England: Robson.

Janesick, V. (1994). The dance of qualitative research design: Metaphor, methodolatry, and meaning. En N. K. Denzin \& Y. Lincoln (Eds.), Handbook of Qualitative Reserach (pp. 209-219). Toushand Oaks, California: SAGE.

Kelly, S. (2008). Understanding the Role of the Football Manager in Britain and Ireland: A Weberian Approach. European Sport Management Quarterly, 8(4), 399-419. https://doi.org/10.1080/16184740802461652

Kelly, S., \& Waddington, I. (2006). Abuse, Intimidation and Violence as Aspects of Managerial Control in Professional Soccer in Britain and Ireland. International Review for the Sociology of Sport, 41(2), 147-164. https://doi.org/10.1177/1012690206075417

King, A. (1997). New directors, customers, and fans: the transformation of English football in the 1990s. Sociology of Sport Journal, 14, 224-240.

https://doi.org/10.1123/ssj.14.3.224 
García-Martí, C.; Durán-González, J., y Gómez-López, M. (2017). El control de la violencia deportiva en el fútbol profesional español (1985-1995). RICYDE. Revista internacional de ciencias del deporte. 50(13), 322-338. https://doi.org/10.5232/ricyde2017.05001

Llopis Goig, R. (2008). Learning and representation: the construction of masculinity in football. An analysis of the situation in Spain. Sport in Society, 11(6), 685-695. https://doi.org/10.1080/17430430802283948

Messner, M. (1988). Sport and Male Domination: The Female Athlete as Contested Ideological Terrain. Sociology of Sport Journal, (5), 197-211. https://doi.org/10.1123/ssj.5.3.197

Messner, M. (1989). Masculinities and Athletic Carrers. Gender and Society, 3(1), 71-88. https://doi.org/10.1177/089124389003001005

Messner, M. (1990). Boyhood, Organized Sports and the Construction of Masculinities. Journal of Contemporary Ethnography, 18(4), 416-444. https://doi.org/10.1177/089124190018004003

Ortí, A. (1996). La apertura y el enfoque cualitativo o estructural: la entrevista abierta semidirectiva y la discusión de grupo. En M. García Ferrando, J. Ibáñez, \& F. Alvira (Eds.), El análisis de la realidad social: métodos y técnicas de investigación (pp. 189221). Madrid: Alianza Editorial.

Parker, A. (1996). Chasing the «Big-Time». Football Apprenticeship in the 1990s. University of Warwick.

Roderick, M. (2003). Work, self and the transformation of identity A sociological study of the careers of professional footballers. University of Leicester.

Roderick, M.; Waddington, I., \& Parker, G. (2000). Playing Hurt: Managing injuries in English Professional Football. International Review for the Sociology of Sport, 35(2), 165-180. https://doi.org/10.1177/101269000035002003

Sandvoss, C. (2003). A game of two halves. Londres: Routledge.

Simón Sanjurjo, J.A. (2014). Fútbol e identidades: la actuación de la selección española de fútbol en los Juegos Olímpicos de Amberes y París a través de su impacto en la prensa. Revista Brasileira de Ciências do Esporte, 36(1), 225-239. https://doi.org/10.1590/S0101-32892014000100015

Valles, M. (2000). Técnicas cualitativas de investigación social. Madrid: Síntesis DL. 